\title{
Antihyperglycemic, hypolipidemic and antioxidant enzymes effect of Strobilanthes crispus juice in normal and streptozotocin-induced diabetic male and female rats
}

\begin{abstract}
The aim of the present study was to investigate the effect of Strobilanthes crispus juice on glucose, lipid profile, glutathione peroxidase and superoxide dismutase in normal and streptozotocin-induced diabetic male and female albino Sprague-Dawley rats. This study was conducted on normal and streptozotocin-induced diabetic male and female Sprague-Dawley rats fed with basal diet and S. crispus juice with different doses 1.0, 1.5 and $2.0 \mathrm{~mL} \mathrm{k-1}$ b.wt. for 30 days. The results showed that significant $(\mathrm{p}<0.05)$ decrease in serum glucose levels in male and female diabetic and normal rats with treated $\mathrm{S}$. crispus juice $(1.0,1.5$ and $2.0 \mathrm{~mL} \mathrm{k}$ 1 b.wt.). Cholesterol and triglyceride level significantly $(\mathrm{p}<0.05)$ decreased in diabetic rats treated with 1.0, 1.5 and $2.0 \mathrm{~mL} \mathrm{k-1}$ b.wt. of S. crispus juice. Cholesterol, triglyceride and LDL-cholesterol level showed reduction in treated male and female normal rats. HDLcholesterol showed the increasing but not significant $(\mathrm{p}<0.05)$ difference in treated diabetic and normal male and female rats. Glutathione peroxidase and superoxide dismutase activities significantly $(\mathrm{p}<0.05)$ increased in treated diabetic and normal male and female rats. In conclusion, S. crispus juice possesses antihyperglycemic, hypolipidemic and antioxidant effect in streptozotocin-induced diabetic rats. Thus, S. crispus juice could be the alternative treatment for lowering glucose, cholesterol and triglyceride for diabetic patients in the future.
\end{abstract}

Keyword: Strobilanthes crispus juice; Glucose; Lipid profile; Antioxidant 